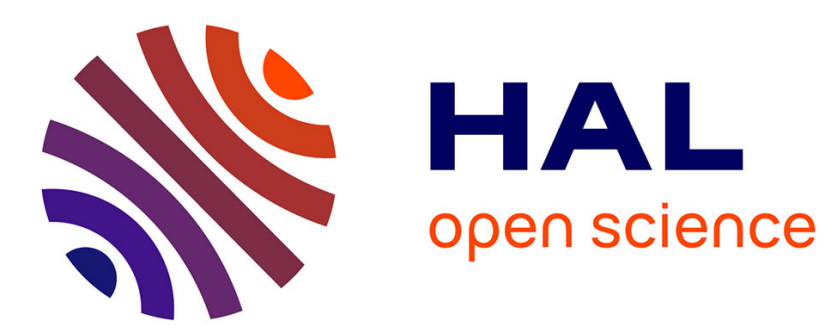

\title{
Torsional Mechanical Oscillator Driven by the Orbital Angular Momentum of Sound
}

Benjamin Sanchez-Padilla, Etienne Brasselet

\section{To cite this version:}

Benjamin Sanchez-Padilla, Etienne Brasselet. Torsional Mechanical Oscillator Driven by the Orbital Angular Momentum of Sound. Physical Review Applied, 2020, 13 (6), 10.1103/PhysRevApplied.13.064069 . hal-02894592

\section{HAL Id: hal-02894592 \\ https://hal.science/hal-02894592}

Submitted on 9 Jul 2020

HAL is a multi-disciplinary open access archive for the deposit and dissemination of scientific research documents, whether they are published or not. The documents may come from teaching and research institutions in France or abroad, or from public or private research centers.
L'archive ouverte pluridisciplinaire HAL, est destinée au dépôt et à la diffusion de documents scientifiques de niveau recherche, publiés ou non, émanant des établissements d'enseignement et de recherche français ou étrangers, des laboratoires publics ou privés. 


\title{
Torsional Mechanical Oscillator Driven by the Orbital Angular Momentum of Sound
}

\author{
Benjamin Sanchez-Padilla $\odot$ and Etienne Brasselet* \\ Univ. Bordeaux, CNRS, Laboratoire Ondes et Matière d'Aquitaine, F-33400 Talence, France
}

(Received 15 April 2020; revised manuscript received 17 May 2020; accepted 26 May 2020; published 29 June 2020)

\begin{abstract}
We demonstrate that the orbital angular momentum of radiating waves can be used to drive a mechanical oscillator, an option that has remained elusive to date on experimental grounds whatever the nature of the waves. This is done using an amplitude-modulated ultrasonic wave interacting with a centimeter-size torsional pendulum. Achieved resonant quantitative measurements of the acoustic radiation torque and material properties set the basis for orbital-angular-momentum-based metrology applications and possibly cooling of the rotational degree of freedom of macroscopic objects.
\end{abstract}

DOI: 10.1103/PhysRevApplied.13.064069

\section{INTRODUCTION}

The torsional pendulum holds a special place in the histories of gravitation, electricity, and magnetism where the measurement of tiny torques has often been involved [1]. One can cite the Cavendish experiment devoted to the determination of the density of the Earth [2]. The Einstein-de Haas experiment demonstrating the mechanical nature of the angular momentum associated with electron spins in a ferromagnet is another famous example [3]. It is also a torsional pendulum that was used to detect and measure mechanically the spin angular momentum of electromagnetic waves in the optical domain $[4,5]$ in the 1930s and years later in the radio domain [6]. Of note, its orbital counterpart was implemented only a few years ago in the radio domain [7] and in the optical domain $[8,9]$. The mechanical manifestation of the orbital angular momentum of sound waves has also been revealed using a torsional pendulum $[10,11]$. The demonstration of a resonant torsional mechanical oscillator driven by wave-matter orbital angular momentum coupling, however, is missing, whatever the nature of the wave. In this work we report its implementation using ultrasonic waves.

Here we start by presenting the design and fabrication of the torsional pendulum in Sec. II followed by the model used to describe its response to an amplitude-modulated acoustic wave in Sec. III. Qualitative observations are reported in Sec. IV using a first design that points out the importance of the choice of the resonant frequency in order to exploit the data using analytical solutions. Finally, a second design enabling a quantitative analysis is demonstrated in Sec. V and metrology applications are presented, such

\footnotetext{
*etienne.brasselet@u-bordeaux.fr
}

as the measurement of the complex shear modulus and the measurement of acoustic radiation torque.

\section{TORSIONAL PENDULUM DESIGN}

Our approach relies on three-dimensionally (3D) printed torsional pendulums made of prototyping resin PR57-K (Autodesk) that are used in the air at atmospheric pressure and room temperature. As sketched in Fig. 1(a), a torsional wire of diameter $D$ and length $L$, which defines the $z$ axis, is fixed at one end and holds a disk of radius $R=15 \mathrm{~mm}$ and height $H=1 \mathrm{~mm}$ at the other end. At the bottom of the disk, a slab with radius $R$ and helical corrugations facing downwards is placed using doublesided tape. The helical surface is designed with $\ell$ identical adjacent angular sectors having azimuthally varying thickness $t(\phi)=\ell h \phi /(2 \pi)$ on the top of a disk pedestal with height $h^{\prime}=1 \mathrm{~mm}$, where $h$ is the step height and $\phi$ is the azimuthal angle in the $(x, y)$ plane.

This design allows an airborne sound wave generated by a flat transducer, with radius $a=5 \mathrm{~mm}$ and placed at a distance $d$ from the helical surface, to exert a torque on the suspended part of the pendulum. Indeed, the large mismatch of acoustic impedance between the air and the resin leads to virtually unitary acoustic reflectivity. Moreover, as the wave is reflected off the helical mirror, its wavefront is twisted and the reflected field thus carries orbital angular momentum directed along the $z$ axis, which is a feature common to "twisted waves" (e.g., light [12], sound [13], gravitation [14], electrons [15], neutrons [16]). The conservation of angular momentum implies that the torsional pendulum acquires angular momentum, also directed along the $z$ axis. Harmonic modulation of the pressure field magnitude is therefore expected to drive the torsional pendulum to resonance when the forcing frequency matches that of the mechanical oscillator, whose natural frequency 


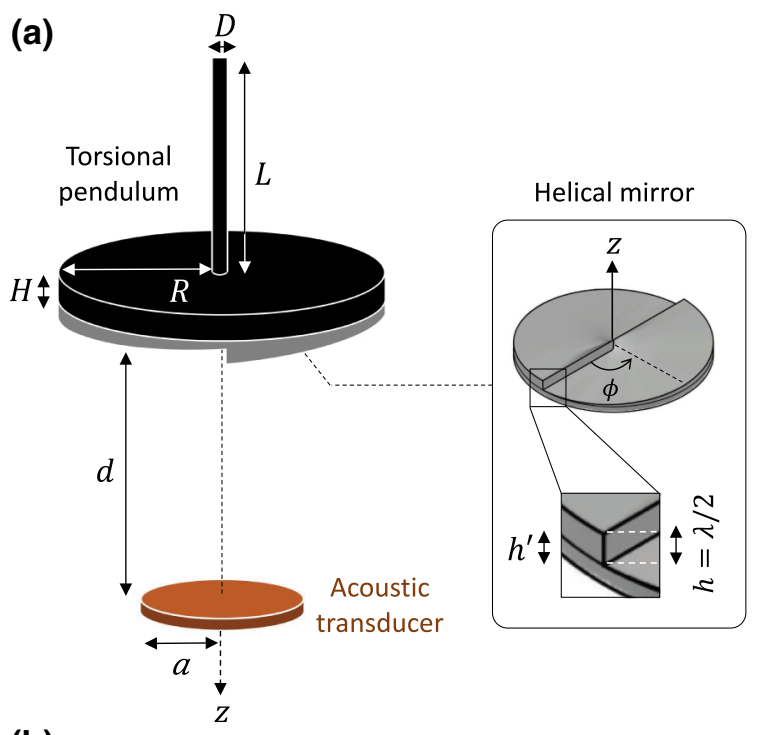

(b)

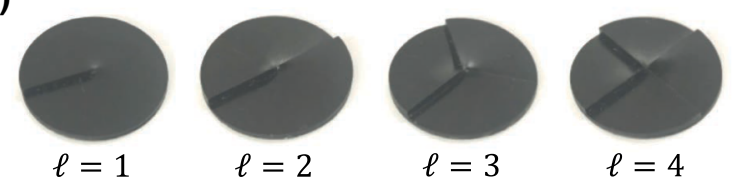

FIG. 1. (a) Geometry of the acoustic torsional pendulum experiment. In all experiments reported in this work we have $a=$ $5 \mathrm{~mm}, R=15 \mathrm{~mm}, H=1 \mathrm{~mm}, h^{\prime}=1 \mathrm{~mm}$, and $h=1.71 \mathrm{~mm}$, and the transducer operates at a nominal output acoustic power $P_{0}$ using a fixed external electrical modulation depth at frequency $F$. The helical mirror inset shows a helically corrugated plate with $\ell=2$. (b) The set of 3D printed helically corrugated plates with topological charges $1 \leq \ell \leq 4$ with $R=15 \mathrm{~mm}, h^{\prime}=$ $1 \mathrm{~mm}$, and $h=1.71 \mathrm{~mm}$.

is $F_{0}=(1 / 2 \pi) \sqrt{K / J}$, where $K>0$ is the torsion constant of the wire and $J$ is the moment of inertia of the suspended part of the pendulum along the $z$ axis.

Here we choose a step height $h=\lambda / 2$ for the suspended helical mirrors, where $\lambda=3.43 \mathrm{~mm}$ is the wavelength in the air associated with the ultrasonic wave frequency $f=100 \mathrm{kHz}$ [see Fig. 1(b)]. This choice implies that the reflected (" $r$ ") pressure field is of the form $p_{r} \propto \exp (-i \omega t+i \kappa z-i \ell \phi)$, where $\omega=2 \pi f$ and $\kappa=2 \pi f / c+i \alpha$ is the complex wave vector, with $c=$ $343 \mathrm{~m} \mathrm{~s}^{-1}$ and $\alpha \simeq 0.38 \mathrm{~m}^{-1}$ at $100 \mathrm{kHz}$ frequency [17] being the sound wave celerity and attenuation in air, respectively. The reflected sound wave thus corresponds to an acoustic vortex beam with topological charge $-\ell$, whose generation is associated (within the paraxial approximation) with an acoustic radiation torque $\Gamma_{z}=\ell P / \omega$ exerted on the helical mirror where $P$ is the acoustic power intercepted by the helical mirror [18].

\section{MODEL}

Noting that $\ell$ and $\omega$ are imposed by the experimental design and that $P>0$, the ideal implementation of a single-frequency driving acoustic torque $\Gamma_{z}=\Gamma_{0}$ $\cos (2 \pi F t)$, where $\Gamma_{0}$ is a constant and $F$ is the driving frequency, is not possible. Instead, we externally drive the amplitude electrical source of the transducer at a frequency $F \ll f$, which leads to an incident (" $i$ ") modulated pressure field of the form $p_{i}(t)=p_{0}[1+$ $\epsilon \cos (2 \pi F t)] \cos (2 \pi f t)$, where $p_{0}$ is a constant and $0<$ $\epsilon<1$ is the modulation depth of the pressure field. Since $P \propto\left\langle p_{i}^{2}\right\rangle$, where the brackets refer to time averaging over one pressure oscillation period, the expression of the driving acoustic torque is written as

$$
\Gamma_{z}(t)=\frac{\ell P}{\omega}\left[1+\frac{\epsilon^{2}}{2}+2 \epsilon \cos (2 \pi F t)+\frac{\epsilon^{2}}{2} \cos (4 \pi F t)\right] .
$$

In the limit of small angular deviation $\theta$ of the suspended part of the pendulum in the plane $(x, y)$, the mechanical response obeys the second-order differential equation

$$
J \frac{d^{2} \theta}{d t^{2}}+\gamma \frac{d \theta}{d t}+K \theta=\Gamma_{z}(t)
$$

where $\gamma>0$ is the damping coefficient and $K>0$ is the torsion constant associated with the real part of the complex shear modulus $\tilde{G}=G^{\prime}+i G^{\prime \prime}$ according to $K=$ $\left(\pi D^{4} / 32 L\right) G^{\prime}$. Since Eq. (2) is linear, the solution to the multiple-frequency external forcing given by Eq. (1) is given by the superposition of the solutions of individual forcing at frequencies $0, F$ and $2 F$. On the one hand, the constant forcing leads to a static angular deviation $\Theta_{\text {stat }}=$ $\left(1+\epsilon^{2} / 2\right) \ell P /(K \omega)$. On the other hand, the harmonic forcing at frequency $v$ leads to a stationary dynamic angular deviation oscillating at the same frequency, with magnitude $\theta_{\text {dyn }}^{(v)}$, whose expression can be modeled noting that the damping has two contributions of distinct origins. Namely, an internal one associated with the imaginary part of the shear modulus of the material constituting the wire and an external one associated with the viscous torque exerted by the air surrounding the pendulum in motion around the $z$ axis. Considering the steady-state dynamics of a harmonic forcing at frequency $F$, the internal contribution is expressed as [19]

$$
\gamma_{\text {int }}=\mathcal{K} /(2 \pi F),
$$

where $\mathcal{K}>0$ is associated with the imaginary part of the complex shear modulus according to $\mathcal{K}=\left(\pi D^{4} / 32 L\right) G^{\prime \prime}$. Neglecting the hydrodynamic contribution associated with the moving wire, whose diameter is much smaller than that of the suspended part of the pendulum, a fair estimate of the external contribution is made from the knowledge of the hydrodynamics of an infinitely think disk immersed in a fluid of density $\rho$ and dynamic viscosity 
$\eta$ that spins at frequency $F$ around its axis of revolution. According to Ovseenko [20], if the Reynolds number $\operatorname{Re}=2 \pi F R^{2} \rho / \eta$ satisfies $\zeta \operatorname{Re}^{2} \ll 1$ with $\zeta \sim 10^{-3}$, the hypothesis of creeping flow is valid and [21]

$$
\gamma_{\mathrm{ext}}=(32 / 3) \eta R^{3} \text {. }
$$

In order to gauge the validity of the latter assumption, we note that all our experiments are made for a resonant oscillating frequency up to $100 \mathrm{~Hz}$, hence $\zeta \operatorname{Re}^{2}$ up to 1 noting that $\rho=1.2 \mathrm{~kg} \mathrm{~m}^{-3}$ and $\eta=2 \times 10^{-5} \mathrm{~Pa}$ s for the air under ambient conditions. Therefore, there is no need to refine the Stokes framework in order to estimate the order of magnitude of $\gamma_{\text {ext }}$. The ratio $\gamma_{\text {int }} / \gamma_{\text {ext }}$ is therefore evaluated from Eqs. (3) and (4) as

$$
\frac{\gamma_{\mathrm{ext}}}{\gamma_{\text {int }}}=\frac{2048}{3} \frac{\eta L R^{3} F}{D^{4} G^{\prime \prime}}
$$

At resonance and using the typical value $G^{\prime \prime}=0.1 \mathrm{GPa}$, this gives $\gamma_{\text {ext }} / \gamma_{\text {int }} \lesssim 10^{-3}$ and $\gamma_{\text {ext }} / \gamma_{\text {int }} \lesssim 10^{-4}$ for the first and second design, respectively, of the pendulum presented in this work. Therefore, keeping only the internal viscous shear losses of the twisted wire, one obtains

$$
\theta_{\mathrm{dyn}}^{(v)}=a_{v} \frac{\ell P}{K \omega}\left[\left(1-\frac{v^{2}}{F_{0}^{2}}\right)^{2}+\left(\frac{\mathcal{K}}{K}\right)^{2}\right]^{-1 / 2},
$$

where $a_{F}=2 \epsilon$ and $a_{2 F}=\epsilon^{2} / 2$. Since $a_{F}>a_{2 F}$, we further focus on the dominant harmonic $v=F$ for which the maximal angular deviation magnitude $\Theta_{\text {dyn }}^{(F)}=2 \epsilon \ell P /(\mathcal{K} \omega)$ is reached at the resonance frequency $F=F_{0}$.

\section{QUALITATIVE EXPERIMENTS}

At first, we design a pendulum with resonant frequency $F_{0} \simeq 10 \mathrm{~Hz}$ enabling a direct demonstration using a camera according to the setup depicted in Fig. 2(a). A laser beam is loosely focused via lens $L_{1}$ onto a $100-\mu \mathrm{m}$-thick mirror having an area of a few square millimeters, on which the beam diameter is approximately $0.5 \mathrm{~mm}$. The reflected light beam is then focused onto a camera placed in the focal plane of the second lens $L_{2}$. The beam spot position $x$ allows monitoring of the angle $\theta=x /\left(2 f_{2}\right)$, where $f_{2}$ is the focal length of $L_{2}$. As the modulation frequency $F$ is increased starting from zero, the amplitude of the periodic displacement of the beam spot increases until it reaches a maximum at $F=F_{0}$. The dynamics of the beam spot at resonance for $\ell=4$ is displayed in Fig. 2(b), which exhibits the expected sinusoidal behavior (see solid curve). This demonstrates qualitatively our main claim: the implementation of a torsional mechanical oscillator driven by the orbital angular momentum of a radiating wave.

Despite that the choice of a small value of $d$ has the advantage of optimizing the intercepted acoustic power

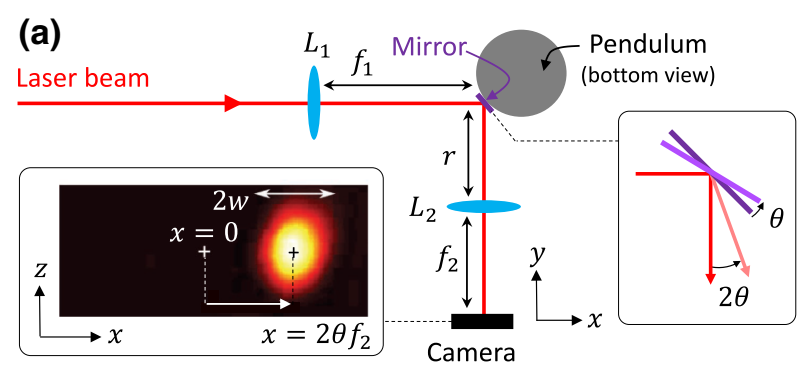

(b)

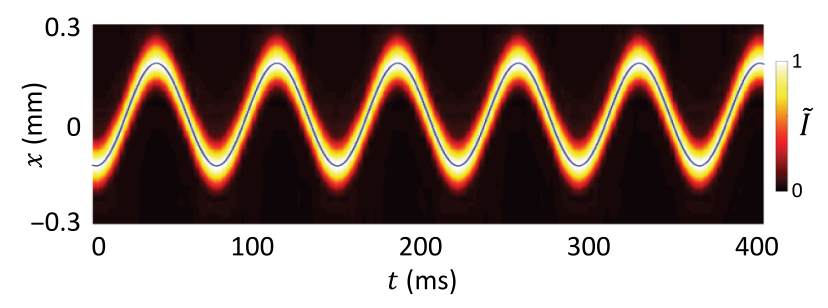

FIG. 2. Acoustic torsional pendulum experiment. (a) Sketch of the experimental setup used for the direct observation of the pendulum dynamics. The light beam is provided by a He$\mathrm{Ne}$ laser operating at a wavelength of $633 \mathrm{~nm}$, the lenses $L_{1}$ and $L_{2}$ have focal lengths $f_{1}=0.5 \mathrm{~m}$ and $f_{2}=1 \mathrm{~m}$, and $r \sim 6 \mathrm{~m}$. (b) Spatiotemporal experimental dynamics of the beam spot intensity profile $I(x, z, t)$ in the plane of the camera, introducing the one-dimensional normalized intensity profile $\tilde{I}(x, t)=\int I(x, z, t) d z / \max _{x}\left[\int I(x, z, t) d z\right]$, for the parameters $\ell=4, d=15 \mathrm{~mm}, L=5 \mathrm{~cm}$, and $D=1 \mathrm{~mm}$. The solid line is a sinusoidal fit.

$P$ and that the choice of a low resonant frequency $F_{0}$ allows for user-friendly direct monitoring of the dynamical motion of the pendulum using a low-frame-rate videoimaging system, this comes with a practical drawback. Indeed, our electric source fails to be driven with a constant $\epsilon$ in the low-frequency regime, which prevents a quantitative unraveling of the universal Lorentzian line shape of the resonant oscillator. The latter issue is ascertained by placing a piezoelectric sensor at a couple of centimeters from the transducer, forming a on-axis acoustic cavity, and adjusting its position in order to maximize the delivered voltage $V(t) \propto p_{i}(t)$ [see Fig. 3(a)]. The modulation depth $\epsilon$ is measured by envelope detection of the voltage signal $V(t)$ delivered by the sensor. The results are shown in Fig. 3(b), which exhibits a strongly frequencydependent $\epsilon$ for $F<50 \mathrm{~Hz}$. Noting that $\epsilon=0.48 \pm 0.01$ between 80 and $120 \mathrm{~Hz}$, we therefore design a second generation of pendulum characterized by a resonant frequency close to $100 \mathrm{~Hz}$, around which the modulation depth can be considered constant.

\section{QUANTITATIVE EXPERIMENTS}

We opt for $L=30 \mathrm{~mm}$ and $D=2.5 \mathrm{~mm}$, and the distance $d$ is substantially increased up to $d=100 \mathrm{~mm}$ in order to eliminate cavity effects between the transducer and 
(a)

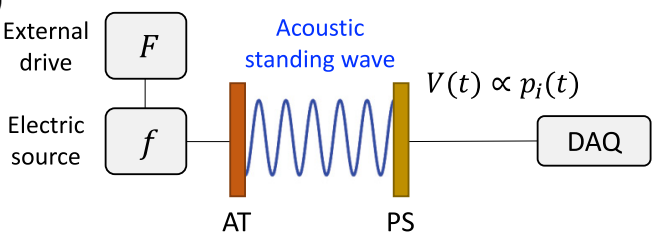

(b)

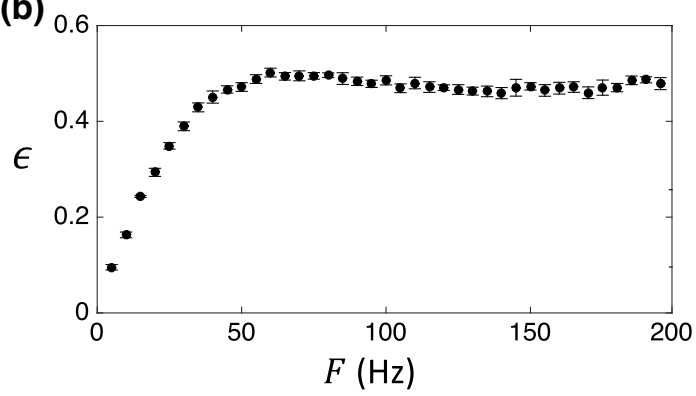

FIG. 3. Modulation depth of the driving pressure field. (a) Sketch of the setup. AT: acoustic transducer; PS: piezoelectric sensor; DAQ: data acquisition system. (b) Experimental modulation depth $\epsilon$ as a function of the modulation frequency $F$ of the pressure field, measured by envelope detection of the voltage signal $V(t)$ delivered by the sensor.

the pendulum (see Appendix A). Experimentally, a stiffer torsional wire and a substantially larger distance from the transducer imply dealing with smaller angular deviations, which prevents accurate video monitoring following the detection framework used in the previous section. This leads us to replace the lens $L_{2}$ and the camera by a lateral effect position sensor [22] placed at a distance $r=6.15 \mathrm{~m}$ from the mirror attached to the pendulum, which gives $\theta=x /(2 r)$, and we use a lens $L_{1}$ with $f_{1}=1 \mathrm{~m}$.

The mechanical resonances are retrieved by sweeping the driving frequency from $F=1$ to $200 \mathrm{~Hz}$ in a linear manner during $10 \mathrm{~min}$ at a sampling frequency of $8.33 \mathrm{kHz}$. Then, wavelet transform processing gives access to $\theta_{\text {dyn }}^{(F)}$ as a function of $F$ (see Appendix B). Obtained results are summarized in Fig. 4(a), which corresponds to the collection of five independent sweeping measurements for each value of $\ell$, for $1 \leq \ell \leq 4$. For each $\ell$, we fit the dataset according to Eq. (6) that is rewritten as

$$
\theta_{\text {dyn }}^{(F)}=\Theta_{\text {dyn }}^{(F)} \frac{G^{\prime \prime}}{G^{\prime}}\left[\left(1-\frac{F^{2}}{F_{0}^{2}}\right)^{2}+\left(\frac{G^{\prime \prime}}{G^{\prime}}\right)^{2}\right]^{-1 / 2},
$$

using $G^{\prime}, G^{\prime \prime}$, and $\Theta_{\text {dyn }}^{(F)}$ as independent adjustable parameters, recalling that $G^{\prime}$ is the only unknown parameter for $F_{0}^{2}=D^{4} G^{\prime} /\left(64 \pi L M R^{2}\right)$, where $M$ is the mass of the suspended part of the pendulum. The Lorentzian line shape is experimentally confirmed whatever $\ell$ (see solid curves). Moreover, all 20 experiments merge into a single universal curve when using normalized quantities for both the angular deviation and the forcing frequency [see Fig. 4(b)],
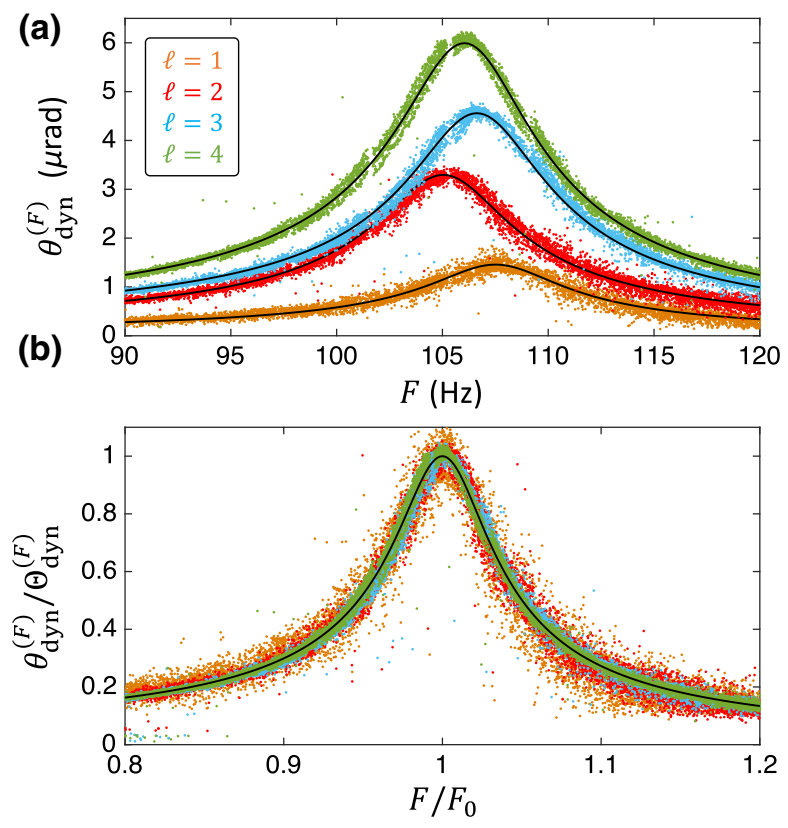

FIG. 4. Resonant mechanical oscillator driven by acoustic orbital angular momentum. (a) Torsional mechanical resonance for four kinds of generated acoustic vortex by the helically corrugated pendulum with $\ell=(1,2,3,4)$. Markers: experimental data of five independent experiments for each value of $\ell$. Solid curves: best-fit adjustment from Eq. (7). (b) Same as in (a) using normalized angular deviation $\theta_{\text {dyn }}^{(F)} / \Theta_{\text {dyn }}^{(F)}$ and normalized frequency $F / F_{0}$. Solid curve refers to the Lorentzian fit of all 20 experiments.

in agreement with Eq. (7). This behavior thus provides a technique to measure the complex shear modulus parameters. This is done by adjusting the data of the normalized resonance curves with $G^{\prime}$ and $G^{\prime \prime}$ as the two adjustable parameters [see solid curve in see Fig. 4(b)]. Namely, we find $G^{\prime}=1.27 \mathrm{GPa}$ and $G^{\prime \prime}=75.9 \mathrm{MPa}$.

These robust experimental observations also allow one to address the challenge of quantitative tests of the acoustic radiation torque formulation in the paraxial regime, so far based on the observation of objects immersed in fluids set into spin owing to dissipative $[23,24]$ or nondissipative [18,25] transfer of orbital angular momentum between wave and matter. Here, the acoustic torque contribution of the harmonic at frequency $F$ is expressed as $\Gamma_{\text {dyn }}^{(F)}=2 \epsilon \ell P / \omega=\mathcal{K} \Theta_{\text {dyn }}^{(F)}$. Its measurement as a function of the topological charge $\ell$ is extracted from the Lorentzian adjustments reported in Fig. 4(a) and the results are displayed in Fig. 5(a), where a linear fit gives the slope $\xi=15.0 \mathrm{nN} \mathrm{m}$.

Testing the theory implies recovering the slope $\xi$ from its expression $\xi=2 \epsilon P / \omega$. This requires one to determine experimentally the acoustic power $P$ intercepted by the pendulum. This is achieved by three independent radiation force balance measurements. It consists of irradiating 

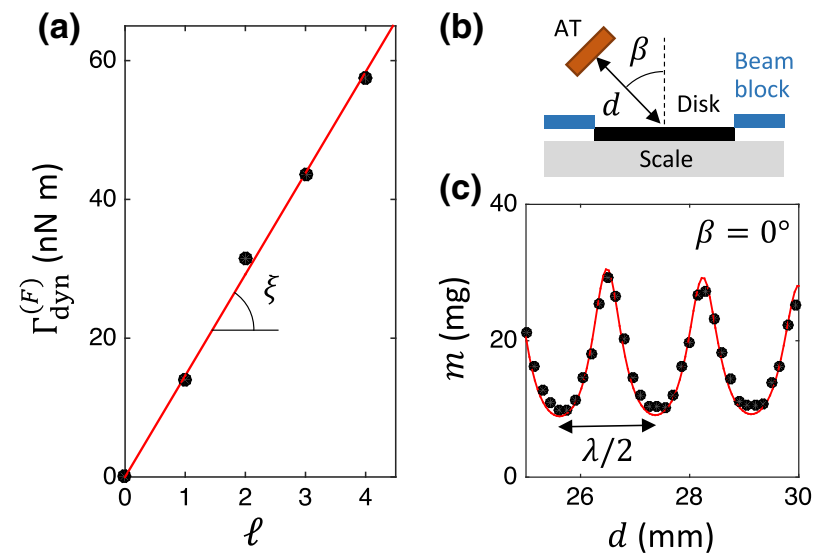

FIG. 5. Quantitative measurement of the acoustic radiation torque. (a) Measurement of the acoustic torque contribution of the harmonic at frequency $F: \Gamma_{\text {dyn }}^{(F)}$ versus the topological charge $\ell$. Solid line is a linear fit. (b) Sketch of the setup for acoustic power measurement from a radiation pressure experiment using a precision scale. AT: acoustic transducer. The 3D printed disk has a 30-mm diameter. (c) Mass measurements as a function of the distance $d$ at normal incidence. Solid line refers to simulations using the total power $P_{0}$ as the single adjustable parameter.

from the air a precision scale at an incidence angle $\beta$ [see Fig. 5(b)] and measuring the mass $m$ resulting from the acoustic radiation force exerted on a two-dimensionally printed disk with a diameter of $30 \mathrm{~mm}$, which behaves as a perfect mirror.

First experiment. We choose $d=25 \mathrm{~mm}$ and $\beta=45^{\circ}$, which gives $P_{0}=25.0 \pm 0.7 \mathrm{~mW}$ via $m g=2 \cos (\beta) P_{0} / c$, where $g$ is the acceleration of gravity. Exploiting the calculated relationship $P \simeq 0.34 P_{0}$ [see Fig. 6(a)], this gives $P=9.3 \mathrm{~mW}$.

Second experiment. We choose a normal incidence $(\beta=$ $0^{\circ}$ ) by retrieving the Fabry-Perot effect as the distance $d$ varies [see Fig. 5(c) and Appendix C]. Adjusting the measured data with the calculations made using the scalar beam propagation method with $P_{0}$ as an adjustable parameter gives $P_{0}=31.6 \mathrm{~mW}$. The relationship $P \simeq 0.34 P_{0}$ gives $P=11.7 \mathrm{~mW}$.

Third experiment. Here we proceed to a direct evaluation of $P$ made at normal incidence and using $d=100 \mathrm{~mm}$ as is the case for the resonant torque measurements. This gives $P=7.5 \pm 0.3 \mathrm{~mW}$ via $m g=2 P / c$.

We thus obtain $\xi=13.6 \pm 2.5 \mathrm{nNm}$ and, recalling the unavoidable imperfections of the helical corrugations (e.g., the measured height step $h$ differs from $\lambda / 2$ up to a few percent and the on-axis geometrical singularity leads to imperfections over a radius of less than $1 \mathrm{~mm}$ ), we conclude to a fair quantitative experimental validation of the paraxial theory for the orbital angular momentum of sound waves.

\section{CONCLUSION}

The proposed torsional mechanical oscillator driven by orbital angular momentum contributes to conceiving rotational metrology techniques such as shear modulus measurements or radiation torque calibration of sources of acoustic orbital angular momentum [26-30]. Noticing that in optics, the mechanical effects based on the interplay between the angular momentum of light and matter attract interest for both cavity and cavityless schemes (e.g., [31]), our results based on acoustic waves might contribute to foster the research field of "angular wave mechanics," for instance . Indeed, by reporting on a rotational oscillator based on acoustic orbital angular momentum, we propose an experimental platform to explore wave-matter interplay in the presence of angular momentum exchanges. Remarkably, dealing with sound waves provides radiation torque with far larger magnitude than that of light as it scales as $\omega^{-1}$, hence making easy the implementation of macroscopic rotational experiments. In particular, the experimental implementation of the cooling of the rotational degree of freedom of macroscopic objects following the scheme proposed by Bhattacharya and Meystre [32] appears conceivable in the acoustic domain.

\section{ACKNOWLEDGMENTS}

The research leading to these results has received funding from CONACYT Mexico.

\section{APPENDIX A}

Here we justify from acoustic propagation simulations that the cavity effects between transducer and pendulum can be neglected for $d=100 \mathrm{~mm}$. The field propagation method used is a generic one that applies to all kinds of scalar propagating waves, which is based on Fourier transforms (see [18]). The latter claim is supported by the results shown in Fig. 6, assuming $\ell=0$ (i.e., no helical corrugation). On the one hand, Fig. 6(a) displays the fraction of the emitted power $P_{0}$ that is intercepted "at first
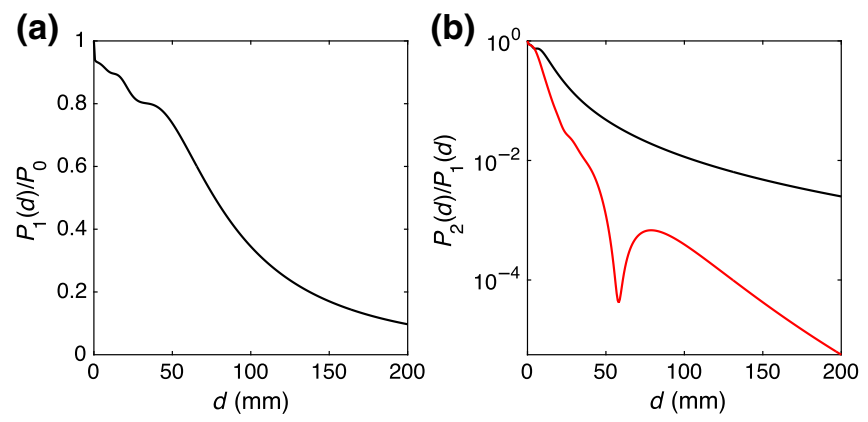

FIG. 6. Simulated dependence of the power fractions $P_{1}(d) / P_{0}$ and $P_{2}(d) / P_{1}(d)$ on the propagation distance. Black curve: $\ell=0$; red curve: $\ell=1$. 
reflection" (subscript "1") by the suspended part of the pendulum, namely $P_{1}(d) / P_{0}$. On the other hand, Fig. 6(b) displays the ratio between the power intercepted "at the second reflection" (subscript " 2 ") and that intercepted "at the first reflection" as a function of $d$, namely $P_{2}(d) / P_{1}(d)$.

Obtained results show that there is approximately $1 \%$ of acoustic power intercepted by the pendulum after one round trip in the cavity for $\ell=0$ [Fig. 6(b), black curve] and this fraction falls to below $0.1 \%$ for $\ell=1$ [Fig. $6(\mathrm{~b})$, red curve]. This value is even smaller as $|\ell|$ increases since the reflected vortex field from the pendulum diffracts into a beam having a doughnut-shaped intensity profile having a dark core whose size increases with $|\ell|$, which drastically increases the cavity losses. We therefore conclude that the cavity effects can be safely discarded at a distance $d=100$ $\mathrm{mm}$ and in that case the intercepted power $P$ equals $P_{1}(d)$ to a good approximation, namely $P \simeq 0.34 P_{0}$.

\section{APPENDIX B}

Here we describe our data-processing protocol, demonstrating its ability to extract the harmonic amplitudes $\theta_{\text {dyn }}^{(\nu)}$ with $v=(F, 2 F)$ from the time-dependent signal $\theta(t)$ recorded by the lateral effect position sensor as the driving frequency $F$ is swept in time. Namely, let us consider the synthetic time series

$$
\theta(t)=\theta_{\text {ideal }}(t)\left[1+\xi_{\sigma}(t)\right]
$$

where $\xi_{\sigma}(t)$ is a normal noise distribution having zero mean value and standard deviation $\sigma$ and

$$
\begin{aligned}
\theta_{\text {ideal }}(t)= & \theta_{\text {stat }}+\theta_{\text {dyn }}^{(F)}(t) \cos [2 \pi F(t) t] \\
& +\theta_{\text {dyn }}^{(2 F)}(t) \cos [4 \pi F(t) t]
\end{aligned}
$$

is the ideal noise-free signal. Mimicking our experimental conditions we take

$$
\begin{gathered}
\theta_{\text {stat }}=A\left(1+\frac{\epsilon^{2}}{2}\right), \\
\theta_{\text {dyn }}^{(F)}(t)=A(2 \epsilon)\left(G^{\prime} / G^{\prime \prime}\right) \Phi^{(F)}(t),
\end{gathered}
$$

and

$$
\theta_{\mathrm{dyn}}^{(2 F)}(t)=A\left(\epsilon^{2} / 2\right)\left(G^{\prime} / G^{\prime \prime}\right) \Phi^{(2 F)}(t),
$$

where $A$ is an unimportant constant factor, here taken as $A=1, \epsilon=1 / 2$, and $G^{\prime \prime} / G^{\prime}=1 / 10$. Moreover,

$$
\Phi^{(F)}(t)=\frac{G^{\prime \prime}}{G^{\prime}}\left[\left(1-\frac{F(t)^{2}}{F_{0}^{2}}\right)^{2}+\left(\frac{G^{\prime \prime}}{G^{\prime}}\right)^{2}\right]^{-1 / 2}
$$

and

$$
\Phi^{(2 F)}(t)=\frac{G^{\prime \prime}}{G^{\prime}}\left[\left(1-\frac{4 F(t)^{2}}{F_{0}^{2}}\right)^{2}+\left(\frac{G^{\prime \prime}}{G^{\prime}}\right)^{2}\right]^{-1 / 2}
$$

are the normalized Lorentzian resonance line shapes of the two harmonics $(F, 2 F)$ and $F_{0}=100 \mathrm{~Hz}$. Also, we choose a driving frequency sweeping defined as $F(t)=$ $f_{\text {start }}+\left(f_{\text {end }}-f_{\text {start }}\right)(t / T)$ with $f_{\text {start }}=1 \mathrm{~Hz}, f_{\text {end }}=200 \mathrm{~Hz}$, and $T=600 \mathrm{~s}$. In addition, we choose a sampling frequency of $5 \mathrm{kHz}$ and a noise level characterized by $\sigma=$ 0.05 .

We use a wavelet transform method from MATLAB software, the continuous wavelet transform built-in function cwt. This function is called $[w v t, f]=\operatorname{cwt}(S, f s)$, where $S$ corresponds to the analyzed signal recorded over a time series given by the vector $\mathrm{T}, \mathrm{fS}$ is the sampling frequency of the signal, and wvt is an $M \times N$ complex matrix, where $M$ and $N$ are the length of the vectors $f$ and $\mathrm{T}$, respectively. The matrix wVt corresponds to the wavelet transform and the vector $\mathrm{f}$ defines the set of discrete frequencies used during the transform. The absolute value of wVt gives the amplitude of the signal at a given frequency and time.

A temporal snapshot of the wavelet transform of the synthetic data is shown in Fig. 7(a) where the labels $v=$ $(F, 2 F)$ refer to the information associated with the first and second harmonic of the signal $\theta(t)$, from which we extract the amplitudes $\theta_{\text {dyn }}^{(v)}(F)$ using the correspondence between time $t$ and driving frequency $F$. The reconstructed resonances are shown in Fig. 7(b) whose Lorentzian fit allows determination of the parameters $\max \left[\theta_{\mathrm{dyn}}^{(v)}\right], F_{0}$, and $G^{\prime \prime} / G^{\prime}$. Obtained results are summarized in Table I,
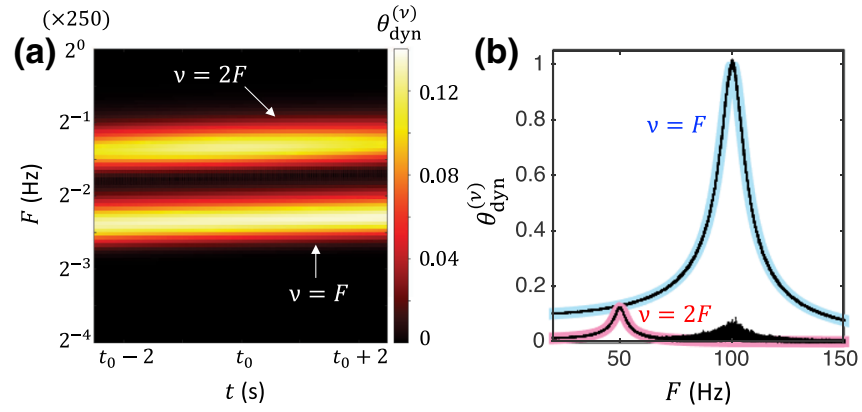

FIG. 7. (a) Wavelet transform of simulated signal in the interval of a few seconds around the time $t_{0}=150 \mathrm{~s}$ at which the instantaneous frequency is $50.75 \mathrm{~Hz}$, hence near the resonance at $F_{0} / 2=50 \mathrm{~Hz}$ for the second harmonic. Amplitude values of the first $(v=F)$ and second $(v=2 F)$ harmonic are extracted as the maximal value at a given time $t$, which corresponds to the frequency $F(t)$. (b) Reconstructed resonances for the first and second harmonic. Black curves: data extracted by wavelet transform; red and blue curves: best fit using Lorentzian line shapes $\theta_{\text {dyn }}^{(v)} \propto \Phi^{(v)}$. 
TABLE I. Obtained values for fit for $\max _{F}\left[\theta_{\mathrm{dyn}}^{(\nu)}\right], F_{0}$, and $G^{\prime \prime} / G^{\prime}$ by fitting the frequency dependence of the amplitude of the first and second harmonic using the Lorentzian resonance line shape.

\begin{tabular}{lccc}
\hline \hline$v$ & $\max _{F}\left[\theta_{\mathrm{dyn}}^{(v)}\right]$ & $F_{0}(\mathrm{~Hz})$ & $G^{\prime \prime} / G^{\prime}$ \\
\hline Synthetic data & & & \\
$F$ & 1 & 100 & $1 / 10$ \\
$2 F$ & $1 / 8$ & 50 & $1 / 10$ \\
Retrieved data & & & \\
$F$ & 0.9971 & 100.00 & 0.0999 \\
$2 F$ & 0.1245 & 100.00 & 0.1002 \\
\hline \hline
\end{tabular}

from which we conclude to a decent reconstruction of the resonant plots.

\section{APPENDIX C}

As stated in the main text, three independent radiation force balance measurements are made in order to determine the intercepted power $P$. One of them consists of measuring the mass $m$ resulting from the acoustic radiation force exerted on a 3D printed disk of $30 \mathrm{~mm}$ in diameter as a function of $25 \mathrm{~mm}<d<30 \mathrm{~mm}$ at normal incidence [see Fig. 5(c)]. The fact that the measured mass oscillates with $d$ emphasizes cavity effects between transducer and flat disk that are both considered as perfect acoustic mirrors in free space. Towards a quantitative description enabling the determination of $P_{0}$ from best-fit adjustment from simulated data, it is important to ensure that a large enough number $N$ of round trips for the wave in the cavity is taken into account. The calculations are made according to the expression

$$
m_{N}(d)=\frac{2 P_{0}}{g c} 2 \pi \int_{0}^{R}\left|\sum_{n=1}^{N} A_{n}(r, d)\right|^{2} r d r
$$

where

$$
\begin{aligned}
& A_{0}(r)=\frac{1}{\pi a^{2}} \operatorname{circ}\left(\frac{r}{a}\right), \\
& A_{1}(r)=\mathcal{P}\left[A_{0}(r) ; d\right],
\end{aligned}
$$

and

$$
A_{n+1}(r)=\mathcal{P}\left[\mathcal{P}\left[A_{n}(r) \operatorname{circ}\left(\frac{r}{R}\right) ; d\right] \operatorname{circ}\left(\frac{r}{a}\right) ; d\right],
$$

with $\operatorname{circ}(x<1)=1$ and $\operatorname{circ}(x>1)=0$, and where $\mathcal{P}[A(r) ; d]$ refers to the propagated axisymmetric wave function $A(r)$ over the distance $d$. The results of calculations are summarized in Fig. 8 for $d=25 \mathrm{~mm}$, from which we decide to fix $N=7$ for the calculation of $m(d)$.

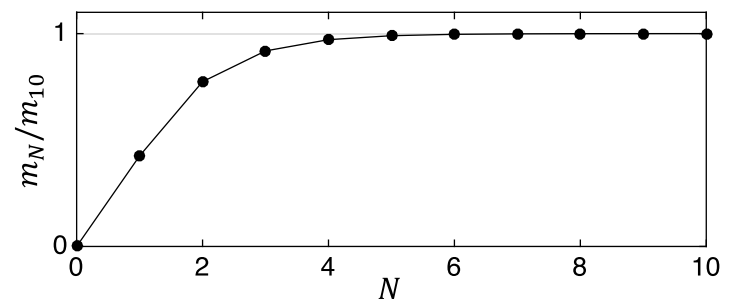

FIG. 8. Simulation of the dependence of the number $N$ of cavity round trips on the measured mass normalized to its limit at large $N$. Calculations are performed at $d=25 \mathrm{~mm}$.

[1] G. Gillies and R. Ritter, Torsion balances, torsion pendulums, and related devices, Rev. Sci. Instrum. 64, 283 (1993).

[2] H. Cavendish, XXI. Experiments to determine the density of the earth, Phil. Trans. R. Soc. Lond. 88, 469 (1798).

[3] A. Einstein and W. De Haas, Experimental proof of the existence of Ampere's molecular currents, Proc. R. Acad. Amsterdam 18, 696 (1915).

[4] R. A. Beth, Mechanical detection and measurement of the angular momentum of light, Phys. Rev. 50, 115 (1936).

[5] A. Holbourn, Angular momentum of circularly polarised light, Nature 137, 31 (1936).

[6] N. Carrara, Torque and angular momentum of centimetre electromagnetic waves, Nature 164, 882 (1949).

[7] O. Emile, C. Brousseau, J. Emile, R. Niemiec, K. Madhjoubi, and B. Thide, Electromagnetically Induced Torque on a Large Ring in the Microwave Range, Phys. Rev. Lett. 112, 053902 (2014).

[8] M. W. Beijersbergen and J. Woerdman, Measuring orbital angular momentum of light with a torsion pendulum, SPIE Proc. 5736, 111 (2005).

[9] O. Emile and J. Emile, Energy, linear momentum, and angular momentum of light: What do we measure? Ann. der Phys. 530, 1800111 (2018).

[10] K. Volke-Sepulveda, A. O. Santillan, and R. R. Boullosa, Transfer of Angular Momentum to Matter from Acoustical Vortices in Free Space, Phys. Rev. Lett. 100, 024302 (2008).

[11] K. D. Skeldon, C. Wilson, M. Edgar, and M. J. Padgett, An acoustic spanner and its associated rotational Doppler shift, New J. Phys. 10, 013018 (2008).

[12] L. Allen, M. W. Beijersbergen, R. J. C. Spreeuw, and J. P. Woerdman, Orbital angular momentum of light and the transformation of Laguerre-Gaussian laser modes, Phys. Rev. A 45, 8185 (1992).

[13] B. T. Hefner and P. L. Marston, An acoustical helicoidal wave transducer with applications for the alignment of ultrasonic and underwater systems, J. Acoust. Soc. Am. 106, 3313 (1999).

[14] I. Bialynicki-Birula and Z. Bialynicka-Birula, Gravitational waves carrying orbital angular momentum, New J. Phys. 18, 023022 (2016).

[15] K. Y. Bliokh, Y. P. Bliokh, S. Savel'Ev, and F. Nori, Semiclassical Dynamics of Electron Wave Packet States with Phase Vortices, Phys. Rev. Lett. 99, 190404 (2007). 
[16] C. W. Clark, R. Barankov, M. G. Huber, M. Arif, D. G. Cory, and D. A. Pushin, Controlling neutron orbital angular momentum, Nature 525, 504 (2015).

[17] H. E. Bass, L. C. Sutherland, A. J. Zuckerwar, D. T. Blackstock, and D. Hester, Atmospheric absorption of sound: Further developments, J. Acoust. Soc. Am. 97, 680 (1995).

[18] B. Sanchez-Padilla, L. Jonusauskas, M. Malinauskas, R. Wunenburger, and E. Brasselet, Direct Mechanical Detection and Measurement of Wave-Matter Orbital Angular Momentum Transfer by Nondissipative Vortex Mode Conversion, Phys. Rev. Lett. 123, 244301 (2019).

[19] N. Tschoegl, Equation of motion of the torsion pendulum and the complex modulus, J. Appl. Phys. 32, 1794 (1961).

[20] Y. G. Ovseenko, Refined formulas for the resistance torque of an ellipsoid of revolution and a circular disk rotating in an infinite incompressible viscous fluid, Fluid Dyn. 4, 100 (1969).

[21] V. G. Levich, Physicochemical Hydrodynamics (PrenticeHall, Englewood Cliffs, NJ, 1962).

[22] The lateral effect position sensor is a silicon photodiodebased pincushion tetra lateral sensor for accurately measuring the displacement of an incident beam relative to the calibrated center (PDP90A, Thorlabs). The sensor is independent of the beam shape and can resolve a minimal displacement of $0.75 \mu \mathrm{m}$ at a wavelength of $635 \mathrm{~nm}$, which corresponds to $0.06 \mu \mathrm{rad}$ with our setup parameters.

[23] C. E. M. Demore, Z. Yang, A. Volovick, S. Cochran, M. P. MacDonald, and G. C. Spalding, Mechanical Evidence of the Orbital Angular Momentum to Energy Ratio of Vortex Beams, Phys. Rev. Lett. 108, 194301 (2012).
[24] A. Anhauser, R. Wunenburger, and E. Brasselet, Acoustic Rotational Manipulation Using Orbital Angular Momentum Transfer, Phys. Rev. Lett. 109, 034301 (2012).

[25] R. Wunenburger, J. I. V. Lozano, and E. Brasselet, Acoustic orbital angular momentum transfer to matter by chiral scattering, New J. Phys. 17, 103022 (2015).

[26] M. Terzi, S. Tsysar, P. Yuldashev, M. Karzova, and O. Sapozhnikov, Generation of a vortex ultrasonic beam with a phase plate with an angular dependence of the thickness, Moscow Univ. Phys. Bull. 72, 61 (2017).

[27] W. Li, M. Ke, S. Peng, F. Liu, C. Qiu, and Z. Liu, Rotational manipulation by acoustic radiation torque of high-order vortex beams generated by an artificial structured plate, Appl. Phys. Lett. 113, 051902 (2018).

[28] N. Jiménez, V. Romero-García, L. M. García-Raffi, F. Camarena, and K. Staliunas, Sharp acoustic vortex focusing by fresnel-spiral zone plates, Appl. Phys. Lett. 112, 204101 (2018).

[29] M. Baudoin, J.-C. Gerbedoen, A. Riaud, O. B. Matar, N. Smagin, and J.-L. Thomas, Folding a focalized acoustical vortex on a flat holographic transducer: Miniaturized selective acoustical tweezers, Sci. Adv. 5, eaav1967 (2019).

[30] R. D. Muelas-Hurtado, J. L. Ealo, and K. Volke-Sepúlveda, Active-spiral fresnel zone plate with tunable focal length for airborne generation of focused acoustic vortices, Appl. Phys. Lett. 116, 114101 (2020).

[31] H. Shi and M. Bhattacharya, Optomechanics based on angular momentum exchange between light and matter, J. Phys. B: Atom. Mol. Opt. Phys. 49, 153001 (2016).

[32] M. Bhattacharya and P. Meystre, Using a LaguerreGaussian Beam to Trap and Cool the Rotational Motion of a Mirror, Phys. Rev. Lett. 99, 153603 (2007). 